\title{
Médecin-chef sous pression
}

\author{
Jürg Bänninger \\ Dr méd., ancien membre du Comité de direction ReMed
}

\section{Le médecin-chef en orthopédie}

Lors de sa première prise de contact téléphonique avec ReMed, notre confrère a simplement demandé à pouvoir «vider son sac». Il raconte qu'au cours de ses sept dernières années d'activité en tant que médecin-chef dans un hôpital régional, il a acquis une grande expérience et développé une solide résilience. Mais les dernières restructurations au sein de l'hôpital et de son service le hantent, car elles ne touchent pas que lui, mais surtout les patients, qui sont le maillon le plus faible. Indigné, il se confie: «'ai 59 ans et je crois qu'il me faut démissionner. En tant que médecin-chef, j'ai fait de nombreuses fois l'impasse sur mes idéaux, mes intérêts, mes valeurs et accepté que mes arguments soient balayés, mais cette fois, je ne peux plus le tolérer, car ces changements impactent directement la qualité des soins.»

\section{La succession était pourtant bien réglée}

«Un médecin-adjoint en orthopédie exerçant depuis longtemps dans cet hôpital a été nommé médecin-chef dans un autre établissement. Notre médecin-chef se réjouit pour lui, mais n'est pas très heureux qu'il parte. Comme il se doit, il anticipe la succession du médecin-adjoint et pense à une cheffe de clinique engagée et motivée qui serait parfaite pour lui succéder.»

\section{Mais la direction de l'hôpital a d'autres plans}

«Tout semblait bien engagé, jusqu'au soir où le seul médecin de la direction (les autres membres viennent du monde économique) est venu me voir pour m'informer qu'en vue de cette succession, il avait été décidé de revoir entièrement tous les contrats des médecinsadjoints: leur salaire fixe serait désormais nettement plus bas avec une part de salaire variable dépendante du chiffre d'affaires. Une solution inacceptable pour cette spécialiste en orthopédie bourrée de talent. La direction de l'hôpital exigeait en outre l'instauration dans tous les services d'un système de médecin agréé et l'extension de la garde à ces derniers. Le médecin membre de la direction a bien cherché à me défendre, mais les raisons financières ont eu raison de tout. Il a ajouté que je pourrais peut-être y trouver des avantages puisque ce système permettrait de m'alléger d'une partie importante de mes responsabilités.

J'étais consterné, car pour moi m'engager et assumer des responsabilités n'est pas une charge, mais fait partie de ma vocation. Ces managers hospitaliers, qui n'y connaissent rien à la médecine et ne s'intéressent

Des économistes sans connaissances médicales ni expérience dans la gestion d'un service hospitalier imposent de plus en plus aux médecins-chefs leurs conditions de travail.

qu'aux chiffres, m'ont tout simplement ôté toute responsabilité et toute influence sur la gestion de mon service sans me consulter au préalable. La direction de l'hôpital m'a ensuite fait parvenir la somme des économies qu'elle comptait réaliser, sans avoir aucune idée des dégâts causés par ce genre de décisions.»

\section{L'impact sur la gestion et le climat de travail est énorme}

«Les conséquences sont vastes. Notre équipe est très motivée et bien rodée et les collaborateurs sont très satisfaits, ce qui se ressent aussi chez les patients, dont le taux de satisfaction est toujours très élevé dans les sondages. Ils apprécient particulièrement le suivi individuel par le médecin responsable. Au fil des années, une excellente et fructueuse collaboration s'est installée avec l'équipe soignante. Un système de médecin agréé mettrait fin à cette étroite collaboration. J'ai tenté à de nombreuses reprises d'en parler avec la direction de l'hôpital, mais je me suis battu en vain.

\section{ReMed est là pour vous}

Avez-vous ou quelqu'un de votre entourage a-t-il besoin d'un soutien professionnel? Dans ce cas, n'hésitez pas à contacter ReMed: le réseau de soutien pour les médecins vous conseillera de manière compétente dans le respect du secret médical. ReMed peut également intervenir dans d'autres situations de crise professionnelles ou personnelles. Cette offre s'adresse aussi aux personnes de l'entourage de médecins. 24 heures sur 24. Un des médecins conseillers se manifeste dans les 72 heures: www. swiss-remed.ch, help[at]swissremed.ch, tél. 0800073633. 


\section{Groupes de parole}

En Suisse romande (Neuchâtel, Lausanne ou Genève), des groupes de parole composés d'au moins quatre médecins intéressés (installés et/ou en formation postgraduée) et animés par un ou deux médecins sont prévus trois ou quatre fois par année. Les réunions sont l'occasion de discuter et d'échanger autour de problèmes de formation, d'organisation du travail, de relation avec les patients ou, selon les intérêts du groupe, d'autres sujets qui posent problème au travail et risquent de démotiver les médecins.

Les personnes intéressées sont priées de prendre contact avec la Dr Carole Weil (dr.c.weil[at]hin.ch) ou le Dr Franco R. Gusberti (frgusberti[at]hin.ch).

Au final, le directeur a même menacé d'organiser tout le service d'orthopédie selon ce système!»

\section{Il est important de savoir qu'on est pas seul}

En tant que réseau de soutien, ReMed a tout d'abord proposé un entretien confidentiel à ce médecin-chef. Et c'est seulement au cours de cet entretien qu'il a réalisé combien cette situation l'affectait depuis longtemps. Pris au sérieux par son interlocuteur de ReMed, qui comprenait parfaitement la situation, notre confrère a compris qu'il n'était pas seul avec ses soucis. Pendant longtemps, il a cherché à protéger son entourage et n'a jamais rien dit. Aujourd'hui, il découvre qu'en parler, ça fait du bien. Dans ce cas précis,
ReMed n'a aucune influence directe, mais le médecinchef a découvert de nouvelles possibilités en ce qui concerne les démarches envers la direction de l'hôpital et la planification de sa propre carrière. De plus, il a aussi appris à trouver une aide ponctuelle auprès d'une personne expérimentée et de confiance. ReMed lui a proposé un coaching adapté à sa situation qu'il peut solliciter quand bon lui semble. Il est désormais conscient que dans sa fonction de médecin-chef, il doit affronter seul les défis, mais qu'il dispose désormais aussi d'une nouvelle possibilité de soutien à explorer.

\section{Intervisions ReMed pour les premiers} répondants et les membres du réseau

En plus du soutien apporté aux médecins qui demandent conseil, ReMed organise depuis 2009 des intervisions régionales pour que les médecins ayant des confrères pour patients puissent échanger leurs expériences. Ces intervisions permettent la mise en réseau et la constitution de groupes de pairs ( 6 à 8 participants, 2 ou 3 rencontres par année) qui abordent ensemble des questions concrètes liées au mentorat, au coaching, au conseil, à la thérapie ou à d'autres aspects (droit, assurances, etc.). N'hésitez pas à nous contacter et à participer à une de nos réunions pour vous faire une idée de notre travail.

Contact et inscription: Dr méd. SabineWerner, membre du Conseil de direction de ReMed, dr.s.werner[at]hin.ch

Prochaines dates en 2019: 28 février, 7 mars, 4 avril, 16 mai, 13 juin, 26 septembre, 14 novembre et 22 novembre, de $14 \mathrm{~h}$ à $18 \mathrm{~h}$ à Zurich. 